\title{
Darstellung von retinalen Neovaskularisationen mit der optischen Kohärenztomografie-Angiografie im Vergleich mit der Fluoreszeinangiografie
}

\section{Visualization of Retinal Neovascularization with Optical Coherence Tomography Angiography in Comparison with Fluorescein Angiography}

\section{Autoren}

Christian Enders ${ }^{1}$, Franziska Baeurle ${ }^{1}$, Gabriele E. Lang ${ }^{1}$, Jens Dreyhaupt ${ }^{2}$, Simon Trick ${ }^{1}$, Adnan Kilani ${ }^{1}$, Gerhard K. Lang ${ }^{1}$, Jens Ulrich Werner ${ }^{1}$

Institute

1 Klinik für Augenheilkunde, Universitätsklinikum Ulm

2 Institut für Epidemiologie und Medizinische Biometrie, Universität Ulm

\section{Schlüsselwörter}

Neovaskularisation, optische KohärenztomografieAngiografie, OCTA, diabetische Retinopathie, PDR

\section{Key words}

neovascularization, PDR, OCTA, optical coherence

tomography angiography, diabetic retinopathy

$$
\text { eingereicht 24.6.2019 }
$$

akzeptiert 15.7.2019

Bibliografie

DOI https://doi.org/10.1055/a-0983-2271

Klin Monatsbl Augenheilkd 2019; 236: 1325-1330 @ Georg

Thieme Verlag KG Stuttgart · New York I ISSN 0023-2165

\section{Korrespondenzadresse}

Dr. Christian Enders

Klinik für Augenheilkunde, Universitätsklinikum Ulm

Prittwitzstraße 43, 89073 Ulm

Tel.: + 49(0)73150059203, Fax: + 49(0)73150059052

christian.enders@uniklinik-ulm.de

\section{ZUSAMMENFASSUNG}

Zielsetzung Vergleichende Darstellung von retinalen papillären (NVD) und nicht papillären (NVE) Neovaskularisationen in der optischen Kohärenztomografie-Angiografie (OCTA) und in der Fluoreszeinangigrafie (FA) bei Patienten mit proliferativer diabetischer Retinopathie.

Material und Methoden 15 konsekutive Patienten wurden in diese Studie aufgenommen. Alle Patienten erhielten eine
OCTA, wobei jeweils ein Scan von $3 \times 3 \mathrm{~mm}$ der „Region of Interest" mit dem ZEISS OCT Cirrus 5000 mit dem AngioPlexModul aufgenommen wurde. Die Größe der Neovaskularisation (NV) wurde manuell in OCTA und FA ermittelt und zwischen den beiden Messmethoden verglichen.

Ergebnisse 20 Augen von 15 Patienten mit proliferativer diabetischer Retinopathie (PDR) mit einem Durchschnittsalter von 57 Jahren wurden eingeschlossen. Die mittlere Größe der NVDs betrug in der OCTA $3,44 \mathrm{~mm}^{2}$ und in der FA $3,75 \mathrm{~mm}^{2}$, die mittlere Größe der NVDs betrug in der OCTA $1,06 \mathrm{~mm}^{2}$ und in der FA $1,54 \mathrm{~mm}^{2}$. Unter Berücksichtigung einer in der FA generell größer gemessenen Fläche zeigte sich insgesamt eine gute Übereinstimmung der beiden Methoden. Schlussfolgerung Es zeigte sich eine gute Übereinstimmung für die Größe der NVs in beiden Untersuchungsmethoden. Die OCTA kann als einfache und nicht invasive Methode zur Darstellung von retinalen Neovaskularisationen die gängige Diagnostik bestätigen.

\section{ABSTRACT}

Objective Comparison of retinal neovascularizations of the disc (NVD) and elsewhere (NVE) in optical coherence tomography angiography (OCTA) and fluorescein angiography (FA) in patients with proliferative diabetic retinopathy.

Materials and Methods 15 consecutive patients were included in this study. All patients received an OCTA with a $3 \times 3 \mathrm{~mm}$ scan of the region of interest with the ZEISS OCT Cirrus 5000 with the AngioPlex module. The size of the neovascularization (NV) was determined manually in OCTA and FA and compared between the two methods.

Results 20 eyes of 15 patients with proliferative diabetic retinopathy with an average age of 57 years were included. The mean size of NVDs was $3.44 \mathrm{~mm}^{2}$ in OCTA and $3.75 \mathrm{~mm}^{2}$ in FA, the mean size of NVDs was $1.06 \mathrm{~mm}^{2}$ in OCTA and 
$1.54 \mathrm{~mm}^{2}$ in FA. Taking into account a generally larger area measured in the FA, the two methods showed good overall agreement.
Conclusion There was a good agreement for the size of the NVs in both methods. OCTA can be used as a simple and non-invasive method to visualize retinal neovascularizations.

\section{Einleitung}

Die diabetische Retinopathie (DR) ist eine der führenden Ursachen für Erblindung im Erwachsenenalter, wobei der Zeitpunkt der Diagnose entscheidend ist, um rechtzeitige Maßnahmen ergreifen zu können, um eine irreversible Visusminderung oder Erblindung zu verhindern [1 - 3]. Die Fluoresceinangiografie (FA) gilt als Goldstandard zur Diagnose und Stadieneinteilung der DR. Mithilfe der FA kann auch die sichere Diagnose des fortgeschrittenen Stadiums der DR, der proliferativen DR (PDR) mit Bildung von retinalen Neovaskularisationen getroffen und eine entsprechende Therapie eingeleitet werden. Die FA ist in der Augenheilkunde weit verbreitet, birgt jedoch auch einige Risiken und hat Limitationen. Für die FA ist eine intravenöse Verabreichung von Fluorescein-Natrium als Kontrastmittel erforderlich, was ein potenzielles Gesundheitsrisiko für den Patienten darstellen kann. Als unerwünschte Nebenwirkungen können nicht nur Übelkeit und Erbrechen, sondern sehr selten auch ein anaphylaktischer Schock mit Lebensgefahr auftreten [4,5].

Die optische Kohärenztomografie-Angiografie (OCTA) ist ein innovatives, nicht invasives Verfahren und erfordert im Gegensatz zur FA keine Anwendung von Fluorescein als Kontrastmittel. Eine OCTA-Aufnahme dauert etwa $10 \mathrm{~s}$. Die FA ist auch im Vergleich zur nicht invasiven OCTA vom Ablauf für die Untersucher und Patienten deutlich aufwendiger und unangenehmer. Zusätzlich können durch die Extravasation des Fluoresceins in der FA Teile der zu beurteilenden retinalen Strukturen maskiert werden und damit eine genaue Diagnostik erschweren.

Die OCTA stellt seit wenigen Jahren eine funktionelle Erweiterung der strukturellen OCT dar und ermöglicht eine nicht invasive, schnelle 3-D-Visualisierung der mikrovaskulären Strukturen von Netzhaut und Aderhaut. Im Prinzip erfasst es die Bewegung von streuenden Partikeln (in erster Linie Erythrozyten) innerhalb von sequenziellen B-Scans der optischen Kohärenztomografie (OCT), die wiederholt an der gleichen Stelle der Netzhaut durchgeführt werden. Änderungen des zeitlichen Kontrasts an einer bestimmten Stelle deuten auf eine Bewegung (Erythrozytenbewegung) und damit auf die Gefäßposition hin. Es konnte bereits nachgewiesen werden, dass die OCTA die genaue Diagnose und Nachsorge von Gefäßerkrankungen am hinteren Augenabschnitt ermöglicht, da wesentliche Erkenntnisse über Morphologie und Perfusionsstatus gleichzeitig gewonnen werden. Dementsprechend hat diese Technologie ein großes Potenzial für die Diagnose und Nachsorge verschiedener vaskulärer retinaler und chorioidaler Erkrankungen wie der diabetischen Retinopathie (DR), bei retinalen Gefäßverschlüssen und altersbezogener Makuladegeneration [610]. Artefakte können wie bei jedem anderen bildgebenden Verfahren auch bei der OCTA auftreten, sind bei korrekter Patientenauswahl aber meist vermeidbar oder klinisch nicht relevant [1113]. Auch bei der Fluoresceinangiografie können Artefakte die Beurteilung erschweren.
Anhand des Nachweises von Neovaskularisationen sowie abhängig von deren Größe werden verschiedene wichtige Therapieentscheidungen getroffen. Um den Einsatz der FA und damit deren Risiken zu minimieren, ist es notwendig, die OCTA auch dahingehend zu evaluieren, ob sie zuverlässig Neovaskularisationen nachweisen kann und ob sich der Nachweis im Vergleich zum Goldstandard FA unterscheidet.

Wir führten daher in dieser Studie eine vergleichende Darstellung von retinalen papillären (NVD) und nicht papillären (NVE) Neovaskularisationen in der OCTA und der FA durch.

\section{Material und Methoden}

Wir nahmen eine vergleichende Querschnittstudie vor, die von der lokalen Ethikkommission genehmigt wurde (Genehmigung durch die Ethikkommission der Universität, Antragsnummer 388/ 15). Jeder Proband stimmte der Teilnahme schriftlich zu, die Studie wurde in Übereinstimmung mit den Grundsätzen der Erklärung von Helsinki durchgeführt.

In dieser Arbeit wurde die Fläche von Neovaskularisationen in kongruenten Aufnahmen desselben Auges von jeweils der Frühphase mit der FA und OCTA in $\mathrm{mm}^{2}$ gemessen und verglichen; NVDs und NVEs wurden dabei getrennt ausgewertet.

Wir schlossen 20 Augen von 15 konsekutiven Patienten mit der Diagnose proliferative diabetische Retinopathie (PDR) ein, die von August 2016 bis Februar 2017 eine OCTA in unserer Klinik erhielten.

Die OCTA wurde mit dem Spectral-Domain-OCT Cirrus 5000 mit dem AngioPlex-Modul, Softwareversion 9.5.1.13585 (Carl Zeiss Meditec, Inc., Dublin, USA) durchgeführt.

Dieses Gerät arbeitet mit 68000 A-Bildern pro Sekunde und ist mit einem Zeilenophthalmoskop ausgestattet. Mit dem FastTrac ${ }^{\text {TM}}$-Eye-Tracking-System können minimale Augenbewegungen verfolgt werden. Zur Bestimmung des Blutflusses auf der Grundlage von zeitlichen Kontrastunterschieden derselben Lokalisation werden in der AngioPlex-OCTA sog. OMAGc-Algorithmen (OMAGc: „Optical Micro Angiography Complex“) verwendet, die sowohl Amplituden- als auch Phasensignale der B-Bilder vergleichen und zu einer hohen Bildqualität beitragen [14].

Wir nutzten für die Studie eine OCTA-Scangröße von $3 \times 3 \mathrm{~mm}$, um die bestmögliche Scanauflösung zu erhalten und wandten diese auf den Bereich der Papille sowie den Bereich der Region der NVEs an. Die manuelle Messung erfolgte in der Segmentierung „Retina Gesamt“ der OCTA, da sich manchmal in der Segmentierung „vitreoretinales Interface“ auf der Netzhaut- oder Papillenoberfläche liegende Neovaskularisation nicht darstellen lassen. Die angiografischen Befunde wurden in der frühvenösen Phase der FA durchgeführt, um eine möglichste geringe Fluoresceinextravasation zu gewährleisten. Dies ist in > Abb. 1 dargestellt. 

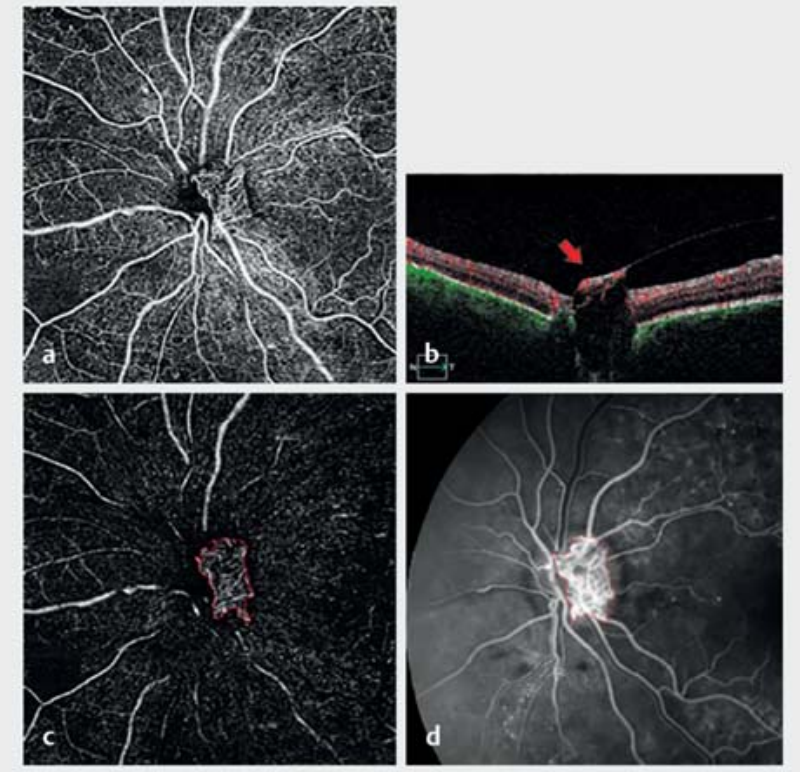

- Abb. 1 Vorgehensweise bei der Auswertung der Neovaskularisationen: a Bild einer NVD in der OCTA-Segmentierung „Retina Gesamt “. b Korrespondierender B-Scan (roter Pfeil) mit der Struktur der Neovaskularisation und Blutflussregistrierung mit roten Punkten farbcodiert dargestellt. c Durch Nachsegmentierung konnte die NVD noch deutlicher abgegrenzt werden, die rote Umrandung zeigt die ausgemessene Fläche. $\mathbf{d}$ Korrespondierender Ausschnitt in der Fluoresceinangiografie in der laminaren Phase mit ebenfalls roter Umrandung der NVD.
Ausschlusskriterien waren signifikante Trübungen der optischen Medien, retinale arterielle oder venöse Verschlüsse, sonstige retinochorioidale Erkrankungen sowie Augenoperation innerhalb der letzten 6 Monate. Die minimale Signalstärke für die OCTA wurde auf 6/10 festgelegt. Die OCTA- und die FA-Bilder wurden von 2 Untersuchern unabhängig voneinander begutachtet.

Dafür wurde für jedes Auge ein relevantes OCTA-en-face-Bild (Segmentierung „Retina Gesamt“) ausgewählt, im B-Scan die Segmentierung überprüft und im Bedarfsfall manuell nachbearbeitet und der Blutfluss in der Neovaskularisation verifiziert. Danach wurde mit Adobe Photoshop (Adobe Photoshop CC 2018 [19.0], Adobe Systems, San José, Kalifornien, USA) das Bild bearbeitet und die Größe der Neovaskularisation in jedem Bild mit Adobe Photoshop-Tools gemessen.

Stetige Variablen wurden durch das arithmetische Mittel, die Standardabweichung, das Minimum und das Maximum beschrieben. Kategoriale Variablen wurden mit absoluten und relativen Häufigkeiten beschrieben. Für den Methodenvergleich der manuellen und der automatisieren Messung wurde der Bland-AltmanPlot verwendet (Vergleich der Größe der Differenz vs. des Mittelwerts beider Methoden) [15-17]. Die mittlere Differenz $d$ ist die fehlende Übereinstimmung zwischen beiden Methoden (Bias). Es ist die Größenordnung des mittleren Unterschiedes zwischen den beiden Methoden. Die 95\%-Grenzen (Limits of Agreement) defi-
- Tab. 1 Demografische Angaben.

\begin{tabular}{|l|l|}
\hline Augen, n & 20 \\
\hline Patienten, n & 15 \\
\hline Geschlecht, n (\%), weiblich/männlich & $6(40) / 9(60)$ \\
\hline Alter, [Jahre] (Mittelwert \pm Standardabweichung) & $56,8 \pm 16,1$ \\
\hline Alter, [Jahre] & $25,1-78,6$ \\
\hline Auge, n (\%), rechts/links & $14(70) / 6(30)$ \\
\hline Art der Neovaskularisation, n (\%), NVD/NVE & $16(66,7) / 8(33,3)$ \\
\hline
\end{tabular}

- Tab. 2 Fläche der NVDs.

\begin{tabular}{|l|l|l|}
\hline Parameter & FA & OCTA \\
\hline $\begin{array}{l}\text { Mittelwert } \pm \text { einfache Standard- } \\
\text { abweichung }\left[\mathrm{mm}^{2}\right]\end{array}$ & $3,75 \pm 4,18$ & $3,44 \pm 3,83$ \\
\hline Werteumfang $\left[\mathrm{mm}^{2}\right]$ & $0,25-15,84$ & $0,27-14,12$ \\
\hline Median $\left[\mathrm{mm}^{2}\right]$ & 2,11 & 1,75 \\
\hline Interquartilsabstand $\left[\mathrm{mm}^{2}\right]$ & 2,82 & 2,55 \\
\hline
\end{tabular}

nieren einen Bereich, der 95\% der Unterschiede zwischen beiden Methoden in der Praxis abdeckt. Die Untersuchung der Übereinstimmung von OCTA und FA wurde weiterhin mittels Streudiagramm untersucht. Bei perfekter Übereinstimmung liegen die Punkte auf der Winkelhalbierenden. Alle statistischen Analysen wurden mit Microsoft Excel V15.32, IBM SPSS Statistics 25 und SAS Version 9.4 (SAS Institute, Chicago, IL, USA) durchgeführt.

\section{Ergebnisse}

Insgesamt konnten 20 Augen von 15 Patienten mit PDR analysiert werden. Sechs dieser Patienten waren weiblich, 9 männlich. Die demografischen Angaben werden in $>$ Tab. 1 dargestellt. Da in manchen Augen sowohl eine NVD als auch eine NVE vorhanden waren, konnten insgesamt 16 NVDs und 8 NVEs ausgewertet werden.

\section{Papilläre Neovaskularisationen}

In der FA betrug die mittlere Fläche der NVDs 3,75 mm². In der OCTA wurde durchschnittlich eine Fläche von $3,44 \mathrm{~mm}^{2}$ gemessen. Alle Angaben sind $>$ Tab. 2, die Einzelwerte dem BlandAltman-Plot in $>$ Abb. 2 zu entnehmen.

Es fällt keine Tendenz und kein Muster der Differenzen auf. Die mittlere Differenz beträgt $-0,31 \mathrm{~mm}^{2}$. Dieser Bias ist als gering einzustufen und unterstützt eine gute Übereinstimmung der Methoden hinsichtlich der NVDs. Die Übereinstimmungsgrenzen liegen bei $-1,40 \mathrm{~mm}^{2}(95 \%-\mathrm{Kl} ;-1,87-0,92)$ als unterer und $0,78 \mathrm{~mm}^{2}(95 \%-\mathrm{Kl} ; 0,31-1,26)$ als oberer Grenze und können in Relation zu den hohen absoluten Maximalwerten ebenfalls als eng betrachtet werden. Auch aus klinischer Sicht sind diese Grenzen akzeptabel. Insgesamt spricht der Bland-Altman-Plot für eine gute Übereinstimmung der Methoden hinsichtlich der NVDs. 


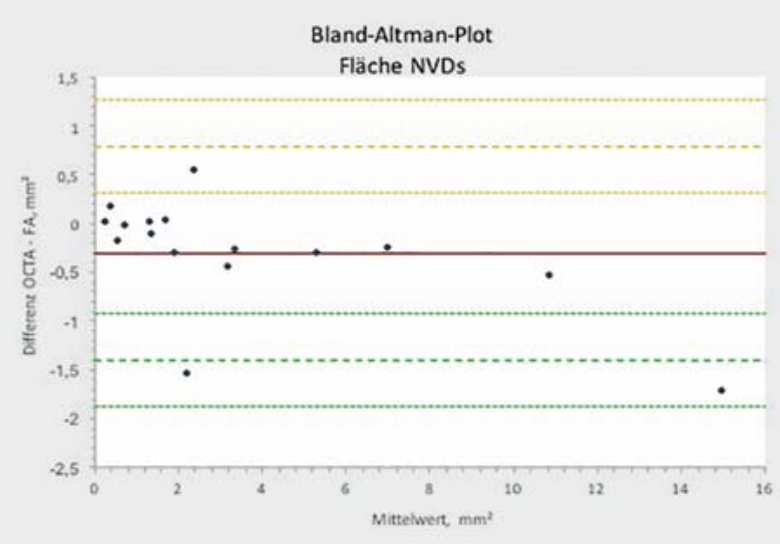

- Abb. 2 Bland-Altman-Plot Fläche NVDs.

- Tab. 3 Fläche der NVEs.

\begin{tabular}{|l|l|l|}
\hline Parameter & FA & OCTA \\
\hline $\begin{array}{l}\text { Mittelwert } \pm \text { Standard- } \\
\text { abweichung }\left[\mathrm{mm}^{2}\right]\end{array}$ & $1,54 \pm 2,56$ & $1,06 \pm 1,62$ \\
\hline Werteumfang $\left[\mathrm{mm}^{2}\right]$ & $0,15-8,16$ & $0,09-5,17$ \\
\hline Median $\left[\mathrm{mm}^{2}\right]$ & 0,33 & 0,38 \\
\hline Interquartilsabstand $\left[\mathrm{mm}^{2}\right]$ & 1,07 & 0,70 \\
\hline
\end{tabular}

\section{Nicht papilläre Neovaskularisationen}

In der FA betrug die mittlere Fläche der NVEs $1,54 \mathrm{~mm}^{2}$. In der OCTA wurde durchschnittlich eine Fläche von $1,06 \mathrm{~mm}^{2}$ gemessen. Alle Angaben sind $>$ Tab. 3, die Einzelwerte dem Streudiagramm in - Abb. $\mathbf{3}$ zu entnehmen. Auf einen Bland-Altman-Plot wurde aufgrund der niedrigen Fallzahl verzichtet.

Das Streudiagramm in $\mathbf{A} \mathbf{A b} \mathbf{b} \mathbf{3}$ legt eine gute Übereinstimmung zwischen FA und OCTA nahe. Bis auf einen Ausreißer liegen alle Punkte nah an der Übereinstimmungsgeraden.

Insgesamt zeigen die Analysen von NVDs und NVEs, dass in der FA größere Flächen gemessen werden als in der OCTA. Die Abweichung ist allerdings in beiden Fällen gering, sodass von einer guten Übereinstimmung von FA und OCTA zur Messung von Neovaskularisationen ausgegangen werden kann.

\section{Diskussion}

Neovaskularisationen sind ein eindeutiges Kennzeichen der PDR [18]. Sie befinden sich meist im Bereich der vitreoretinalen Grenzfläche und tragen als potenzielle Ursache von Glaskörperblutungen oder Netzhautablösungen wesentlich zu Sehverschlechterung oder Erblindung bei Diabetikern bei $[19,20]$. Es ist zur Erstdiagnose und für die Verlaufskontrolle von Patienten mit PDR wichtig, alle Neovaskularisationen zuverlässig beurteilen zu können. Die Einteilung des Stadiums der PDR mit folgender Therapiekonsequenz hängt nicht nur von der Lokalisation, sondern auch von der Größe einer NV ab [21].

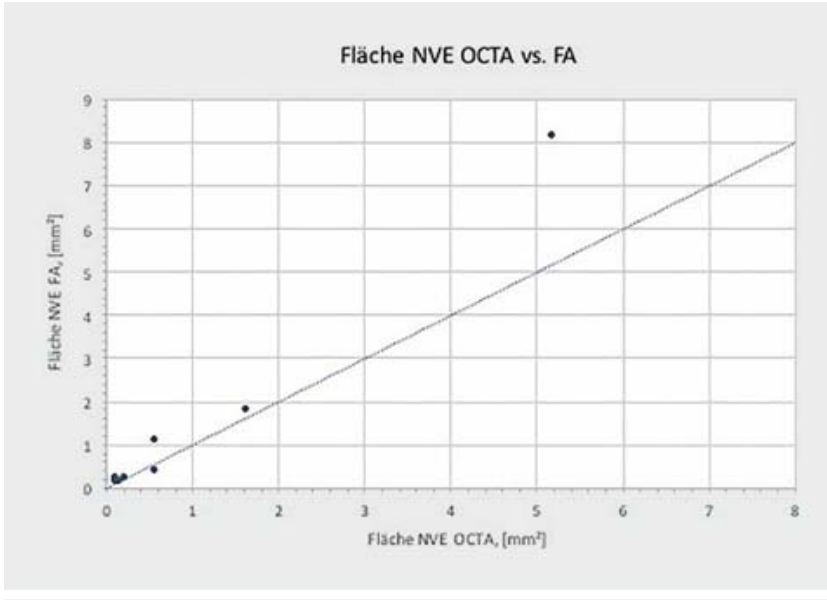

- Abb. 3 Fläche NVE OCTA vs. FA.

Mehrere vorangegangene Studien konnten bereits zeigen, dass die OCTA Neovaskularisationen zuverlässig und die neovaskulären Gefäße in größerer Detailliertheit darstellen kann als die FA [6, 19, 22 -24]. Diese ist dadurch begründet, dass die nicht eliminierbare Leckage von Fluorescein, die den Neovaskularisationen durch ihre defekte Blut-Retina-Schranke eigen ist, durch Maskierung der Gefäßstruktur die Flächenbestimmung in ihrer Genauigkeit eingeschränkt.

Alle Neovaskularisationen konnten an gleicher Stelle auf der Netzhaut in beiden Methoden identifiziert werden. Sowohl für die NVDs als auch für die NVEs ergaben sich in der FA durchschnittlich höhere Flächenwerte als in der OCTA. Dies war im Rahmen des Messverfahrens zu erwarten, da zur Flächenmessung in der FA die neovaskulären Gefäße und die Grenze der Leckage um eine Neovaskularisation herangezogen wurden. Diese Grenze liegt weiter außen als die tatsächlichen kapillaren Grenzen der Neovaskularisation, sodass in der FA durchschnittlich größere Flächen resultieren. Unter Berücksichtigung dieses Faktors stimmen die Werte gut miteinander überein und auch die genauere Analyse mittels Streudiagramm bzw. Bland-Altman-Plot attestierte eine gute Übereinstimmung der Methoden hinsichtlich der Neovaskularisationen. Zusätzlich fiel bei der Auswertung der Angiogramme auf, mit welcher Detailliertheit die OCTA Gefäße zeigt und im Vergleich zur FA in der Lage ist, Neovaskularisationen 3-dimensional darzustellen. Während in der FA vor allem die Leckage das Vorhandensein einer Neovaskularisation andeutet, ihre eigentliche Struktur aber oft verdeckt, stellt die OCTA die Gefäße in hoher Auflösung dar.

Ein weiterer Vergleich der Fläche von Neovaskularisationen zwischen FA und OCTA wurde in der aktuellen Literatur nicht gefunden, sodass die Ergebnisse dieser Arbeit bislang allein stehen. Weitere prospektive Studien, die einen solchen quantitativen Flächenvergleich durchführen, sind nötig, um diese Ergebnisse einordnen zu können. Diese Arbeit unterstützt die These, dass die OCTA in der Lage ist, Neovaskularisationen in Übereinstimmung mit der FA sehr zuverlässig und mitunter detaillierter abzubilden [19, 22,24]. Durch die größere Genauigkeit der Darstellung in der OCTA können neue Erkenntnisse hinsichtlich der Pathologie 
von Neovaskularisationen gewonnen werden und auch nicht invasiv Therapiekontrollen nach Laser oder Anti-VEGF-Therapie durchgeführt werden, um die Inaktivierung der Neovaskularisationen im Verlauf zu beurteilen und die Indikation für eine Nachbehandlung zu stellen. Ishibazawa und Kollegen konnten zeigen, dass die unterschiedliche Morphologie der Neovaskularisationen auch Hinweise auf die Aktivität der PDR geben kann [19]. So spricht das Vorhandensein von unregelmäßigen, kleinkalibrigen Kapillaren auf den Rändern größerer Gefäßaussprossungen für eine aktive Proliferation, während unter Therapie ein Rückgang dieser Ausläufer zu beobachten ist. Aufgrund der Leckage ist die FA i.d.R. nicht in der Lage, solche Feinheiten darzustellen, sodass die OCTA sich als wertvolles Instrument in der Evaluation von Neovaskularisationen und deren Aktivität erweist.

Eine Einschränkung, die die OCTA in dieser Hinsicht allerdings aufweist, sind Schwierigkeiten bei der Segmentierung [25]. Da Neovaskularisationen sich im vitreoretinalen Interface befinden [20] oder anderweitig die vom Gerät festgesetzten Segmentierungsgrenzen überschreiten, liefert die automatische Segmentierung teilweise unzureichende Ergebnisse und es müssen immer die B-Scans kontrolliert und ggf. manuell nachsegmentiert werden, um vorhandene Neovaskularisationen nicht zu übersehen oder in ihrer Ausdehnung falsch einzuschätzen. Manche Anteile der Neovaskularisationen, die weit in den Glaskörperraum hineinwachsen, können evtl. gar nicht von der OCTA erfasst werden, da sie deren Scantiefe überschreiten, sodass sich nur ein Schatten darstellt [26]. Diese Gefahr birgt die FA nicht, da jede Neovaskularisation unabhängig von deren Tiefe i.d.R. schnell durch die Leckage identifiziert werden kann. Zudem wurde gezeigt, dass im Rahmen der DR auch Neovaskularisationen einen verlangsamten Blutfluss aufweisen [27], sodass wie für die Mikroaneurysmen in der OCTA die Gefahr besteht, unter die nötige Schwelle zur Signalerfassung zu fallen und damit der Bildgebung zu entgehen.

Trotz der guten Übereinstimmung zwischen FA und OCTA hinsichtlich der Flächen empfiehlt sich derzeit also ein komplementärer Einsatz beider Methoden zur Beurteilung von Neovaskularisationen. Während die FA in der Lage ist, Neovaskularisationen mithilfe der leicht erkennbaren, hyperfluoreszenten Leckage eindeutig zu identifizieren, kann die OCTA durch ihre detaillierte Darstellung helfen, diese in Ausdehnung und Form präziser zu definieren und damit wichtige Zusatzinformationen für Therapie und Verlaufskontrolle des Patienten zu liefern. Derzeit ist die Fläche, die mit der OCTA untersucht werden kann, auch zu klein im Vergleich zur Weitwinkel-FA, um sicher alle Neovaskularisationen im Bereich der Netzhaut zu erfassen, da sie bis mittelperipher oder selten auch peripher auftreten können. Papilläre Neovaskularisationen lassen sich gut erkennen.

Als Limitationen unserer explorativen Studie sind die manuelle Vorgehensweise bei der Flächenmessung zu sehen sowie die relativ geringe Fallzahl. Weitere prospektive Studien mit größeren Fallzahlen sind nötig, um die quantitative Übereinstimmung zwischen FA und OCTA hinsichtlich von Neovaskularisationen zu bestätigen und den Nutzen bei der Therapiekontrolle zu überprüfen.

\section{Interessenkonflikt}

[1] Lee R, Wong TY, Sabanayagam C. Epidemiology of diabetic retinopathy, diabetic macular edema and related vision loss. Eye Vis (Lond) 2015; 2: 17. doi:10.1186/s40662-015-0026-2

[2] Ting DSW, Cheung GCM, Wong TY. Diabetic retinopathy: global prevalence, major risk factors, screening practices and public health challenges: a review. Clin Exp Ophthalmol 2016; 44: 260-277. doi:10.1111/ ceo.12696

[3] Yau JW, Rogers SL, Kawasaki R et al. Global prevalence and major risk factors of diabetic retinopathy. Diabetes Care 2012; 35: 556-564. doi:10.2337/dc11-1909

[4] Dithmar S, Holz FG. Fluoreszenzangiographie in der Augenheilkunde. Heidelberg: Springer Medizin; 2008

[5] Kwiterovich KA, Maguire MG, Murphy RP et al. Frequency of adverse systemic reactions after fluorescein angiography: results of a prospective study. Ophthalmology 1991; 98: 1139-1142

[6] Lang GE, Enders C, Werner JU. [New possibilities in retinal diagnostics using OCT angiography]. Klin Monatsbl Augenheilkd 2016; 233: 613-621. doi:10.1055/s-0042-105325

[7] De Carlo TE, Romano A, Waheed NK et al. A review of optical coherence tomography angiography (OCTA). Int J Retina Vitreous 2015; 1: 5. doi:10.1186/s40942-015-0005-8

[8] Yu S, Lu J, Cao D et al. The role of optical coherence tomography angiography in fundus vascular abnormalities. BMC Ophthalmol 2016; 16: 107. doi:10.1186/s12886-016-0277-2

[9] Enders C, Baeuerle F, Lang GE et al. Comparison between findings in optical coherence tomography angiography and in fluorescein angiography in patients with diabetic retinopathy. Ophthalmologica 2019. doi:10.1159/000499114

[10] Werner JU, Böhm F, Lang GE et al. Comparison of foveal avascular zone between optical coherence tomography angiography and fluorescein angiography in patients with retinal vein occlusion. PloS One 2019; 14 : e0217849. doi:10.1371/journal.pone.0217849

[11] Enders C, Lang GE, Dreyhaupt J et al. Quantity and quality of image artifacts in optical coherence tomography angiography. PloS One 2019; 14 : e0210505. doi:10.1371/journal.pone.0210505

[12] Spaide RF, Fujimoto JG, Waheed NK. Image artifacts in optical coherence tomography angiography. Retina 2015; 35: 2163-2180. doi:10.1097| IAE.0000000000000765

[13] Falavarjani KG, Al-Sheikh M, Akil H et al. Image artefacts in swept-source optical coherence tomography angiography. Br J Ophthalmol 2017; 101 : 564-568. doi:10.1136/bjophthalmol-2016-309104

[14] Zhang A, Zhang Q, Chen CL. Methods and algorithms for optical coherence tomography-based angiography: a review and comparison. J Biomed Opt 2015; 20: 100901. doi:10.1117/1.JBO.20.10.100901

[15] Bunce C. Correlation, agreement, and Bland-Altman analysis: statistical analysis of method comparison studies. Am J Ophthalmol 2009; 148: 46. doi:10.1016/j.ajo.2008.09.032

[16] McAlinden C, Khadka J, Pesudovs K. Statistical methods for conducting agreement (comparison of clinical tests) and precision (repeatability or reproducibility) studies in optometry and ophthalmology. Ophthalmic Physiol Opt 2011; 31: 330-338. doi:10.1111/j.1475-1313.2011.00851.x

[17] Wilkinson CP, Ferris FL, Klein RE et al. Global Diabetic Retinopathy Project Group. Proposed international clinical diabetic retinopathy and diabetic macular edema disease severity scales. Ophthalmology 2003; 110: 1677-1682

[18] Lang GK, Hrsg. Augenheilkunde. 5. Aufl. Stuttgart: Thieme; 2014: 235238

[19] Ishibazawa A, Nagaoka T, Yokota H. Characteristics of retinal neovascularization in proliferative diabetic retinopathy imaged by optical coher-

Die Autoren geben an, dass kein Interessenkonflikt besteht. 
ence tomography angiography. Invest Ophthalmol Vis Sci 2016; 57: 6247-6255. doi:10.1167/iovs.16-20210

[20] Schmidt-Erfurth U. Netzhaut, Aderhaut und Glaskörper. In: Augustin A], Hrsg. Augenheilkunde. 3. Aufl. Heidelberg, New York, Tokio: Springer; 2007: 360-367

[21] [Anonymous]. Grading diabetic retinopathy from stereoscopic color fundus photographs - an extension of the modified Airlie House classification: ETDRS report number 10. Early Treatment Diabetic Retinopathy Study Research Group. Ophthalmology 1991; 98: 786-806

[22] De Carlo TE, Bonini Filho MA, Baumal CR et al. Evaluation of preretinal neovascularization in proliferative diabetic retinopathy using optical coherence tomography angiography. Ophthalmic Surg Lasers Imaging Retina 2016; 47: 115-119. doi:10.3928/23258160-20160126-03
[23] Khadamy J, Aghdam KA, Falavarjani KG. An update on optical coherence tomography angiography in diabetic retinopathy. J Ophthalmic Vis Res 2018; 13: 487-497. doi:10.4103/jovr.jovr_57_18

[24] Stanga PE, Papayannis A, Tsamis E et al. New findings in diabetic maculopathy and proliferative disease by swept-source optical coherence tomography angiography. Dev Ophthalmol 2016; 56: 113-121. doi:10.1159/000442802

[25] Lang GE, Enders C, Loidl M. Präzise Befundung mit der OCT-Angiografie Artefakte erkennen und ausschließen. Klin Monatsbl Augenheilkd 2017; 234: 1154-1160. doi:10.1055/s-0043-118224

[26] Hwang TS, Jia Y, Gao SS. Optical coherence tomography angiography features of diabetic retinopathy. Retina 2015; 35: 2371-2376

[27] Spaide RF, Fujimoto JG, Waheed NK et al. Optical coherence tomography angiography 2018. Prog Retin Eye Res 2018; 64: 1-55. doi:10.1016/j. preteyeres.2017.11.003 